Canad. J. Math. Vol. 73 (3), 2021 pp. 717-736

http://dx.doi.org/10.4153/S0008414X20000139

(c) Canadian Mathematical Society 2020. This is an Open Access article, distributed

under the terms of the Creative Commons Attribution licence (http://creativecommons.org/licenses/ by/4.0/), which permits unrestricted re-use, distribution, and reproduction in any medium, provided the original work is properly cited.

\title{
Extension Property and Universal Sets
}

\author{
Łukasz Kosiński and Włodzimierz Zwonek
}

\begin{abstract}
Motivated by works on extension sets in standard domains, we introduce a notion of the Carathéodory set that seems better suited for the methods used in proofs of results on characterization of extension sets. A special stress is put on a class of two-dimensional submanifolds in the tridisc that not only turns out to be Carathéodory but also provides examples of two-dimensional domains for which the celebrated Lempert Theorem holds. Additionally, a recently introduced notion of universal sets for the Carathéodory extremal problem is studied and new results on domains admitting (no) finite universal sets are given.
\end{abstract}

\section{Extension Property and Carathéodory Sets}

\subsection{Introduction and State of Affairs}

For a set $V \subset D$, where $D$ is a domain in $\mathbb{C}^{n}$, we denote by $\mathcal{O}(V)$ the set of holomorphic functions defined on $V$ as the set of all $f: V \rightarrow \mathbb{C}$ such that for arbitrary $w \in V$, there are an open neighborhood $W$ of $w$ in $D$ and a holomorphic $g: W \rightarrow \mathbb{C}$ such that $f$ coincides with $g$ on $V \cap W$. By $H^{\infty}(V)$, we mean the algebra of bounded holomorphic functions on $V$. In what follows, many results could be formulated and proved for any algebras of holomorphic functions on $V$ containing polynomials; however, we restrict ourselves to the special case of the algebra of bounded holomorphic functions. Additionally, for the simplicity of formulations and clarity of presentation, we always assume that the set $V$ has analytic structure in the sense that $V$ is always to be assumed to be an analytic set in the given domain $D$. This means that $V$ is relatively closed in $D$, and for every point $x \in V \subset D$, there exist an open set $U \subset D$ containing $x$, and $f_{1}, \ldots, f_{m} \in \mathcal{O}(U)$ such that $V \cap U=\left\{z \in U: f_{j}(z)=0, j=1, \ldots, m\right\}$.

The analytic set $V \subset D$ has the extension property if for any $f \in H^{\infty}(V)$, there is an $F \in H^{\infty}(D)$ such that $F \equiv f$ on $V$ and $\|F\|_{D}=\|f\|_{V}$.

The origin of the problem of the existence of norm preserving extensions of bounded holomorphic functions goes back to Rudin's book ([22, Theorem 7.5.5]). The key step in that area of research can be found in [4] where the problem was solved for the bidisc. More precisely, the following result was proved.

Theorem 1.1 (see [4]) Let $V$ be a relatively polynomially convex subset of $\mathbb{D}^{2}$. Then $V$ has the extension property if and only if it is a retract.

Received by the editors November 17, 2019; revised February 5, 2020.

Published online on Cambridge Core February 24, 2020.

The first author is partially supported by NCN grant SONATA BIS no. 2017/26/E/ST1/00723. The second author is partially supported by the OPUS grant no. 2015/17/B/ST1/00996 of the National Science Centre, Poland.

AMS subject classification: 32D15, 32F45.

Keywords: extension set, Carathéodory set, Lempert theorem, universal set for the Carathéodory extremal problem. 
Recall that $V \subset D$ is a retract if there is a holomorphic map $r: D \rightarrow D$ such that its range is $V$ and $r_{\mid V}$ is the identity. It is an obvious observation that any retract has the extension property.

Later, Kosiński and McCarthy proved, relying on the Lempert theory, that the same statement as above holds for the class of two-dimensional strictly convex domains $D$ (see [15]). They also showed some necessary form of the sets with the extension property in sufficiently smooth strongly linearly convex domains in higher dimensions. Such sets must be totally geodesic.

On the other hand, in the paper [2], the authors described the sets with the extension property in the symmetrized bidisc and found out that in this case there are sets with the extension property that are not retracts.

Although the problem of the characterization of the extension sets in the simplest case of the polydisc has been studied, it is very frustrating that only some partial results on that topic have been obtained. In this context let us mention results in $[6,10,14,20]$.

The situation in the tridisc $\mathbb{D}^{3}$ was studied in [14].

As one looks at the proofs of results describing the extension sets in a series of papers in quite different situations, a weaker form of the extension property is more natural to work with. Namely, the existence of norm preserving extensions of some extremal functions is essential. And in principle, the results on the description of extension sets just mentioned can be generalized to that new notion. This is formally done in Section 2, where the proofs are given in a detailed and partially novel way in the bidisc only; in other cases, they are merely outlined.

Studying the tridisc $\mathbb{D}^{3}$, the authors showed that one-dimensional sets with the extension property are precisely retracts (that was later generalized to arbitrary polydiscs in [20]), and for two-dimensional sets with the extension property they found a necessary condition ([14, Theorem 6.1]). The last form was, as indicated in [14], not sufficient for the set to have the extension property. Then authors considered a class of two-dimensional subsets that are uniqueness varieties for three dimensional and non-degenerate 3-point Pick interpolation problem in $\mathbb{D}^{3}$ (see [14, Remark 7.4] and [13]). In our paper, we will show that these two-dimensional subsets do satisfy our new notion, but they are not retracts. This is surprising as such a phenomenon occurs neither in the bidisc nor in domains studied in the literature so far. Therefore, this class of two-dimensional algebraic subsets is one of the objects that attracts our attention. More precisely, we look at analytic submanifolds $M_{\alpha}$ defined as the sets

$$
\left\{\left(z_{1}, z_{2}, z_{3}\right) \in \mathbb{D}^{3}: \alpha_{1} z_{1}+\alpha_{2} z_{2}+\alpha_{3} z_{3}=\bar{\alpha}_{1} z_{2} z_{3}+\bar{\alpha}_{2} z_{1} z_{3}+\bar{\alpha}_{3} z_{1} z_{2}\right\},
$$

where $\alpha_{1}, \alpha_{2}, \alpha_{3} \in \mathbb{C}$ are not all zeros.

Remark 1.2 (i) Recall that a characterization of retracts in the polydisc as the sets being graphs of holomorphic functions over lower dimensional polydiscs comes from [11]. Note that if $\alpha_{3} \neq 0$, the surface $M_{\alpha}$ can be written as a graph of a function given by the formula

$$
z_{3}=\psi\left(z_{1}, z_{2}\right)=\omega \frac{a z_{1}+b z_{2}-z_{1} z_{2}}{\bar{b} z_{1}+\bar{a} z_{2}-1}
$$


where $a=\alpha_{1} / \bar{\alpha}_{3}, b=\alpha_{2} / \bar{\alpha}_{3}$, and $\omega=\bar{\alpha}_{3} / \alpha_{3}$. In particular, $M_{\alpha}$ is a graph of a function over the first two variables from $\mathbb{D}^{2}$ if and only if $|a|+|b| \leq 1$, that is, $\left|\alpha_{1}\right|+\left|\alpha_{2}\right| \leq\left|\alpha_{3}\right|$. Therefore, the above-mentioned result of Heath and Suffridge [11] implies that the variety $M_{\alpha}$ is not a retract of the tridisc exactly when $\left|\alpha_{i_{1}}\right|+\left|\alpha_{i_{2}}\right|>\left|\alpha_{i_{3}}\right|$ for all possible permutations $\left(i_{1}, i_{2}, i_{3}\right)$ of the set $\{1,2,3\}$-we shall say that such a triple satisfies the triangle inequality.

(ii) The family $\left\{M_{\alpha}\right\}$ is stable under automorphisms of $\mathbb{D}^{3}$ in the following sense: if $z \in M_{\alpha}$ and $m \in \operatorname{Aut}\left(\mathbb{D}^{3}\right)$ maps $z$ to 0 , then $m\left(M_{\alpha}\right)=M_{\beta}$ for some $\beta$. To prove this, it is enough to consider the case $\alpha_{3} \neq 0$ (permute the coordinates, if necessary). Let us represent $M_{\alpha}$ as in (1.1). Note that the function $\psi$ is inner in the following sense: $\left|\psi\left(z_{1}, z_{2}\right)\right|=1$ for almost all $z_{1}, z_{2}$ in the unit circle. Trivial computations show that $m$ transforms $M_{\alpha}$ to a surface of the form

$$
z_{3}=\varphi\left(z_{1}, z_{2}\right):=\frac{A z_{1}+B z_{2}+C z_{1} z_{2}}{D z_{1}+E z_{2}+F}
$$

where $F \neq 0$. It can be assumed that $F=1$. It is also simple to observe (properties of $m)$ that $\varphi$ is inner. This fact is crucial for the rest of our reasoning.

The following observation is trivial: if

$$
\lambda \longmapsto \frac{\gamma_{1} \lambda+\gamma_{2}}{1+\gamma_{3} \lambda}
$$

is inner (that is maps almost all points from the unit cirle to the unit circle), then $\gamma_{3}=\gamma_{1} \bar{\gamma}_{2}$ and either $\left|\gamma_{1}\right|=1$ or $\left|\gamma_{2}\right|=1$.

Let us apply this observation to $\lambda \mapsto \varphi(\lambda, \omega \lambda)$, where $\omega$ is a unimodular constant (almost arbitrary). If $\gamma_{1}$ appearing in (1.2) is unimodular, we get that $|C|=1$ and

$$
\varphi\left(z_{1}, z_{2}\right)=C \frac{\bar{E} z_{1}+\bar{D} z_{2}+z_{1} z_{2}}{D z_{1}+E z_{2}+1} .
$$

If, in turn, $\left|\gamma_{2}\right|=1$, then either $B$ is unimodular and $\varphi\left(z_{1}, z_{2}\right)=B z_{2}$ or $A$ is unimodular and $\varphi\left(z_{1}, z_{2}\right)=A z_{1}$. Certainly the last two cases cannot occur (otherwise, $M_{\alpha}$ would be of the form $z_{3}=\omega_{j} z_{j}$ for some $\left.j=1,2\right)$. In particular, $m\left(M_{\alpha}\right)$ is of the form (1.3) and consequently can be written as $M_{\beta}$ for $\beta=(c \bar{E}, c \bar{D},-\bar{c})$, where $c^{2}=C$.

Note also that $\beta$ satisfies the triangle inequality if and only if $\alpha$ does. This is an immediate consequence of the fact that $m$ takes holomorphic retracts to holomorphic retracts and the previously mentioned description of such sets.

(iii) All the results for $M_{\alpha}$ presented below are non-trivial precisely when the triple $\alpha$ satisfies the triangle inequality; otherwise, the sets are biholomorphic to the bidisc.

The notion that is new and is basic in our paper is that of an (infinitesimally) Carathéodory set to be defined below. To define the objects we use notion of the Carathéodory pseudodistance (Carathéodory-Reiffen pseudometric) that is an example of a holomorphically invariant function. Since basic properties of holomorphically invariant functions are essential for us, we ask the reader to consult the book [12] on the fundamental properties of these functions.

By $\rho$ we denote the hyperbolic metric on the unit disc $\mathbb{D}:=\{\lambda \in \mathbb{C}:|\lambda|<1\}$. We also denote $\mathbb{T}:=\partial \mathbb{D}$. 
We define the Carathéodory pseudodistance

$$
c_{V}(z, w):=\sup \{\rho(F(z), F(w)): F \in \mathcal{O}(V, \mathbb{D})\} .
$$

Its infinitesimal version, the Reiffen-Carathéodory pseudometric, is defined below

$$
\gamma_{V}(w ; X):=\sup \left\{\left|F^{\prime}(w) X\right|: F \in H^{\infty}(V),\|F\| \leq 1, F(w)=0\right\},
$$

where $w \in V$ is a regular point ( $w \in V_{\text {reg }}$ ), and $X$ is an arbitrary vector from the tangent space $T_{w} V$.

A function $F$ for which the supremum in the definition above is attained is called extremal (resp. infinitesimally extremal) for the pair $(z, w)(\operatorname{resp} .(w ; X))$.

Note that if $V$ is an analytic set in $D$, then $c_{V}(w, z) \geq c_{D}(w, z)$ for any $w, z \in V$ and $\gamma_{V}(w ; X) \geq \gamma_{D}(w ; X)$ for any $w \in V_{\text {reg }}, X \in T_{w} V$.

Definition 1.3 Let $V$ be a set (an analytic variety) in a subdomain $D$ of $\mathbb{C}^{n}$.

We say that $V$ is a Carathéodory set if

$$
c_{D}(z, w)=c_{V}(z, w) \quad \text { for all } z, w \in V .
$$

We say that $V$ is an infinitesimal Carathéodory set if

$$
\gamma_{D}(w ; X)=\gamma_{V}(w ; X)
$$

for any regular point $w \in V_{\text {reg }}$ and $X \in T_{w} V$.

As we have already announced, the (infinitesimal) Carathéodory sets are the ones that admit the norm preserving extensions of (infinitesimally) extremal functions.

It is an elementary observation that if $V$ has the extension property, then it is a Carathéodory set. Any Carathéodory set is an infinitesimal Carathéodory set. In Section 2, we briefly sketch how the known proofs on results describing extension sets presented above apply to the situation of the Carathéodory sets. Note here that any Carathéodory set must be connected.

\subsection{Link to the Lempert Theory}

While trying to characterize the sets having the extension property (or Carathéodory sets), the impact of the Lempert theory of holomorphically invariant functions in convex domains turns out to be essential. First recall that for the domain $D$ and points $w, z \in D$, we define the Lempert function

$$
l_{D}(w, z):=\inf \{\rho(0, t): \exists f \in \mathcal{O}(\mathbb{D}, D) \text { such that } f(0)=w, f(t)=z\} .
$$

Note that the definition of the Lempert function can be easily extended to arbitrary subsets $M \subset \mathbb{C}^{n}$ (with $D$ replaced by $M$ ). In case there is no holomorphic mapping joining $w, z$ lying entirely in $M$ we define $l_{M}(w, z):=\infty$. We can also define the infinitesimal version of the Lempert function. We define

$$
\kappa_{V}(w ; X):=\inf \left\{|\lambda|: \exists f: \mathbb{D} \rightarrow V, f(0)=w, \lambda f^{\prime}(0)=X\right\},
$$

where $w \in V_{\text {reg }}$ and $X \in T_{w} V$. The function $\kappa_{V}$ is called the Kobayashi-Royden pseudometric. 
We call a holomorphic mapping $f: \mathbb{D} \rightarrow D$ a complex geodesic if there is a holomorphic function $F: D \rightarrow \mathbb{D}$ such that $F \circ f$ is an automorphism of the unit disc. In particular, for any $\lambda, \mu \in \mathbb{D}$ we have the equality $\rho(\lambda, \mu)=c_{D}(f(\lambda), f(\mu)), \lambda$, $\mu \in \mathbb{D}$. We call the function $F$ to be the left inverse of the complex geodesic $f$. We also say that the complex geodesic $f$ passes through $w, z \in D$ (resp. $\left.(w ; X) \in D \times \mathbb{C}^{n}\right)$ if $w, z \in f(\mathbb{D})$ (resp. $w=f(\lambda)$ and $X$ is parallel to $f^{\prime}(\lambda)$ for some $\lambda \in \mathbb{D}$ ).

The main result in the Lempert theory is the following.

Theorem 1.4 (see $[18,19]$ ) Let $D$ be a convex domain in $\mathbb{C}^{n}$. Then $c_{D} \equiv l_{D}$. Moreover, if $D$ is also bounded, then for any two points $w, z \in D$ (resp. $\left.w \in D, X \in \mathbb{C}^{n}\right)$, we can find a complex geodesic $f: \mathbb{D} \rightarrow D$ passing through $w, z$ (resp. $(w ; X))$.

Actually, for taut domains $D \subset \mathbb{C}^{n}$ (i.e., such that any sequence of holomorphic mappings $f_{k}: \mathbb{D} \rightarrow D$ is a normal family), the fact that $c_{D}$ coincides with $l_{D}$ is equivalent to the existence for any pair of points $w, z \in D$ of a complex geodesic passing through them. It is quite natural to call any taut domain $D$ such that $c_{D} \equiv l_{D}$ a Lempert domain. The Lempert theorem states that any bounded convex domain is a Lempert domain. Note that the complex geodesics in $D$ are proper holomorphic embeddings of the unit disc into the domain $D$.

Definition 1.5 Let $V$ be an analytic subset of a domain $D$ in $\mathbb{C}^{n}$.

We say that $V$ is totally geodesic in $D$ if for any $z, w \in V, z \neq w$ there exists a complex geodesic $f: \mathbb{D} \rightarrow D$ passing through $z$ and $w$ that lies entirely in $V$.

We say that an analytic set $V$ in $D$ is infinitesimally totally geodesic if for any $w \in V_{\text {reg }}$ and any $X \in T_{w} V$, we find a complex geodesic $f: \mathbb{D} \rightarrow D$ such that $f(0)=w$, the vector $X$ is parallel to $f^{\prime}(0)$ and the image of $f$ lies entirely in $V$.

Proposition 1.6 Any (infinitesimally) totally geodesic set $V$ in a Lempert domain $D$ is an (infinitesimally) Carathéodory set.

Proof Choose distinct points $z, w \in D$ and a holomorphic function $F: V \rightarrow \mathbb{D}$. Let $f$ be a complex geodesic passing through $z, w$ that lies in $V$. Choose $\lambda, \mu \in \mathbb{D}$ so that $f(\lambda)=z, f(\mu)=w$. Then, by the Schwarz lemma,

$$
\rho(F(z), F(w))=\rho\left(F \left(f(\lambda), F(f(\mu)) \leq \rho(\lambda, \mu)=l_{D}(z, w)=c_{D}(z, w) .\right.\right.
$$

Consequently, $c_{V}(z, w) \leq c_{D}(z, w)$, which finishes the proof in the first case. The proof in the infinitesimal case goes along the same lines so we skip it.

\subsection{Main Results}

As we already announced the following theorem is one of the main results of our paper (see Theorem 3.1): the set $M_{\alpha}=\left\{z \in \mathbb{D}^{3}: \alpha_{1} z_{1}+\alpha_{2} z_{2}+\alpha_{3} z_{3}=\bar{\alpha}_{3} z_{1} z_{2}+\bar{\alpha}_{2} z_{1} z_{3}+\bar{\alpha}_{1} z_{2} z_{3}\right\}$ is Carathéodory. Moreover,

$$
l_{M_{\alpha}} \equiv c_{M_{\alpha}} \equiv\left(c_{\mathbb{D}^{3}}\right)_{\mid M_{\alpha}^{2}} .
$$

Although we do not know whether the sets $M_{\alpha}$ are always extension sets, the above result gives not only a new insight into the understanding of the extension property 
and extends results of [14] but also provides an interesting class of Lempert domains. To make the last statement clear we introduce a class of two-dimensional subdomains in $\mathbb{D}^{2}$. For $a, b>0$, we define

$$
D_{a, b}:=\left\{z \in \mathbb{D}^{2}:\left|F_{a, b}\left(z_{1}, z_{2}\right)\right|<1\right\},
$$

where

$$
F_{a, b}\left(z_{1}, z_{2}\right):=\frac{a z_{1}+b z_{2}-z_{1} z_{2}}{a z_{2}+b z_{1}-1} .
$$

Note that the function $F_{a, b}$ is the one that gives a solution $z_{3}\left(z_{1}, z_{2}\right)$ of the equation defining $M_{\alpha}$ for suitably chosen $\alpha$. Similarly as above the domain $D_{a, b}$ is interesting precisely when the triple $\{a, b, 1\}$ satisfies the triangle inequality.

A direct consequence of the above result is the following (see Theorem 3.6): the domain $D_{a, b}$ is a Lempert domain.

As we shall see later, the domains $D_{a, b}$ are, under some obvious assumptions on $a$ and $b$, even not linearly convex (see Remark 3.7). The existence of such a class of domains is interesting from the point of view of the Lempert theory, as the only domain with all holomorphically invariant functions equal for which that equality could not be concluded from the Lempert theorem is the tetrablock (introduced in [1]); see [8]. Recall that the symmetrized bidisc was, at the time of its discovery, the first example posessing such a phenomenon (see $[5,7])$. It turned out later that this domain can be exhausted by strongly linearly convex domains, which made it possible to deduce the equality of all holomorphically invariant functions on the symmetrized bidisc from the Lempert theorem (see [21]).

In Section 4, motivated by a recent paper [2], we consider the universal sets for the Carathéodory problem, i.e., the sets $\mathcal{C} \subset \mathcal{O}(D, \mathbb{D})$, which can replace the set $\mathcal{O}(D, \mathbb{D})$ in the definition of the Carathéodory pseudodistance of the domain $D$. We remark that in dimension one under very mild and natural assumptions, the existence of a finite universal set for the Carathéodory problem implies that the domain is the disc (Theorem 4.3).

It is noted in [2] that in dimension two the existence of a universal set with two elements requires the domain to be the bidisc. We generalize this result showing that the existence of a finite universal set lets the domain embed in the polydisc (possible of higher dimension); see Theorem 4.4. Additionally, the domains $D_{a, b} \subset \mathbb{C}^{2}$ turn out to be examples of the ones admitting three and not two elements in the universal set. Therefore, the situation in dimension two differs from that in dimension one and there are other than the bidisc nice and non-trivial domains admitting a finite number of elements in the universal set; see Example 4.5.

In Section 2, the main stress is put on extension and simplification of the situation in the bidisc, i.e., Theorem 1.1, which is the content of Theorem 2.3 and Corollary 2.4. The characterization of Carathéodory sets in the situation of strictly convex, strongly linearly convex and the symmetrized bidisc is sketched only as the methods from $[3,14,15]$ apply in the general case word by word.

In Section 5, we briefly discuss the problem of a possible structure of universal sets for the Carathéodory extremal problem in the Euclidean ball $\mathbb{B}_{n}$ and we show how to produce universal sets that are "smaller" than the ones obtained in the most evident way. 


\section{Carathéodory Sets Replace Extension Sets}

This section studies connection between Carathéodory and extension sets. Its first aim is to describe Carathéodory sets in the bidisc showing that they are holomorphic retracts. It is thus trivial that both notions coincide there, which, in particular, proves and extends [4]. After a more detailed study of the case of the bidisc, we sketch how the proofs in $[3,14,15]$ can be applied to get the results describing Carathéodory sets in the cases of strictly convex, strongly linearly convex domains and the symmetrized bidisc-the proofs of (formally stronger) results follow exactly the same lines as in appropriate papers.

Proposition 2.1 Let $D$ be a domain in $\mathbb{C}^{n}$ and $V \subset D$ an analytic set. Assume that $c_{V}(z, w)=c_{D}(z, w)>0$ for some $z, w \in V\left(\right.$ resp. $\gamma_{D}(z ; X)=\gamma_{V}(z ; X)>0$, for some $z \in V_{\text {reg }}$ and $X \in T_{z} V$ ). If the function $F: D \rightarrow \mathbb{D}$ is extremal for $(z, w)$ (resp. for $(z ; X)$ ), then $F(V)$ is dense in $\mathbb{D}$.

Proof Suppose that $F(V)$ is not dense in $\mathbb{D}$. Let $\varnothing \neq \bar{\triangle} \subset \mathbb{D}, \bar{\triangle} \cap F(V)=\varnothing$, be a closed disc. In the first case, the result follows from [3, Lemma 9.3], which gives the existence of a holomorphic function $\beta: \mathbb{D} \backslash \bar{\Delta} \rightarrow \mathbb{D}$ such that

$$
\rho(F(z), F(w))<\rho(\beta(F(z)), \beta(F(w))) \leq c_{D}(z, w),
$$

which contradicts the extremality of $F$. The infinitesimal case follows the same idea (and makes use of the same [3, Lemma 9.3]).

Below, we use the notion of balanced points introduced in [4]. A pair $(z ; w) \epsilon$ $\mathbb{D}^{2} \times \mathbb{D}^{2}, z \neq w$ (resp. $\left.(z ; X) \in \mathbb{D}^{2} \times \mathbb{C}^{2}, X \neq(0,0)\right)$ is called balanced (resp. infinitesimally balanced) if it satisfies the equality $\rho\left(z_{1}, w_{1}\right)=\rho\left(z_{2}, w_{2}\right)$ (resp. $\gamma_{\mathbb{D}}\left(z_{1} ; X_{1}\right)=$ $\left.\gamma_{\mathbb{D}}\left(z_{2} ; X_{2}\right)\right)$. Note that being (infinitesimally) balanced is invariant under holomorphic automorphisms of $\mathbb{D}^{2}$ in the sense that if the pair $(z, w)$ (resp. $\left.(z ; X)\right)$ is (infinitesimally) balanced, then so is $\left(a(z) ; a^{\prime}(z)(X)\right)$ (resp. $\left.(a(z), a(w))\right)$ for any automorphism $a$ of $\mathbb{D}^{2}$. It follows from the Schwarz lemma that the (infinitesimally) balanced pair determines uniquely a complex geodesic passing through them-in this case both components of the geodesic are automorphisms of the unit disc).

Lemma 2.2 Let $V \subset \mathbb{D}^{2}$ be polynomially convex analytic subvariety. If there is a balanced pair $(z, w)$ such that $z, w \in V$ and $c_{V}(z, w)=c_{\mathbb{D}^{2}}(z, w)$ (resp. infinitesimally balanced pair $(z, X) \in V_{\text {reg }} \times T_{z} V$ and $\left.\gamma_{V}(z ; X)=\gamma_{\mathbb{D}^{2}}(z ; X)\right)$, then $V$ contains the unique complex geodesic passing through them.

Proof The proof is standard, we are recalling it for the sake of completeness. Losing no generality, we can assume that $z=(0,0)$. Since $((0,0), w)$ (resp. $((0,0) ; X))$ forms a balanced (resp. infinitesimally balanced) pair we get that $w_{2}=\omega w_{1}\left(\right.$ resp. $\left.X_{2}=\omega X_{1}\right)$, $|\omega|=1$. Then making use of the fact that the function $\frac{z_{1}+\bar{\omega} z_{2}}{2}$ is (infinitesimally) extremal for $((0,0), w)$ (resp. $((0,0) ; X))$, due to Proposition 2.1 we get that the set $\left\{\frac{z_{1}+\bar{\omega} z_{2}}{2}: z \in V\right\}$ is dense in $\mathbb{D}$, so the polynomial convexity of $V$ implies that $\{(\lambda, \omega \lambda)$ : $\lambda \in \mathbb{D}\} \subset V$, which finishes the proof. 
Theorem 2.3 Let $V$ be an analytic subvariety of $\mathbb{D}^{2}$ that is polynomially convex. Then $V$ is an infinitesimal Carathéodory set if and only if it is a union of a discrete set and complex geodesics.

Proof The only difficult part is to show that any one-dimensional irreducible component $W$ of an infinitesimal Carathéodory set is (equivalently, contains) a complex geodesic.

The case when there is a point $(z ; X) \in W_{\text {reg }} \times\left(T_{z} W \backslash\{0\}\right)$ that is infinitesimally balanced follows from Lemma 2.2.

Suppose that no pair $(z ; X) \in W_{\text {reg }} \times\left(T_{z} W \backslash\{0\}\right)$ is balanced. Then without loss of generality, we can assume that

$$
\gamma_{\mathbb{D}}\left(z_{2}, X_{2}\right)<\gamma_{\mathbb{D}}\left(z_{1}, X_{1}\right) \text { for every } z \in W_{\text {reg }} \text { and } X \in T_{z}(W) \backslash\{0\} \text {. }
$$

In particular, near every such $z$ the variety $W$ is a graph of a holomorphic function over the first coordinate. Moreover,

$$
\rho\left(z_{2}, w_{2}\right)<\rho\left(z_{1}, w_{1}\right)
$$

if $z, w \in W_{\text {reg }}, z \neq w$ are close enough to each other.

We shall prove that $W$ contains a graph of a holomorphic function $f: \mathbb{D} \rightarrow \mathbb{D}$ with the property $|f(\lambda)|<|\lambda|, \lambda \in \mathbb{D} \backslash\{0\}$ which would finish the proof.

First, note that near $(0,0)$, we get the existence of a holomorphic $f_{0}: \triangle(0, \epsilon) \rightarrow \mathbb{D}$ with the property $\left|f_{0}(\lambda)\right|<|\lambda|, \lambda \in \triangle(0, \epsilon) \backslash\{0\}$ and $\left\{\left(\lambda, f_{0}(\lambda)\right): \lambda \in \triangle(0, \epsilon)\right\}=W \cap$ $\triangle(0, \epsilon)^{2}$. Below, we show how we can extend the function to the whole unit disc. Denote $K:=\pi_{1}\left(\left(W \backslash W_{\text {reg }}\right) \cap\left\{\left|z_{2}\right| \leq\left|z_{1}\right|\right\}\right)$, which is a discrete subset of $\mathbb{D} \backslash \triangle(0, \epsilon)$.

Define $\mathcal{F}$ as the family of all pairs $(U, g)$ where $\Delta(0, \epsilon) \subset U \subset \mathbb{D}, U$ is star-shaped (with respect to 0 ), $g: U \rightarrow \mathbb{D}$ is holomorphic with $|g(\lambda)|<|\lambda|, \lambda \in U \backslash\{0\}$ and $\left.g\right|_{\triangle} \equiv$ $f_{0}$. The identity principle shows that for any two pairs $\left(U_{j}, g_{j}\right) \in \mathcal{F}$ we have $g_{1}=g_{2}$ on $U_{1} \cap U_{2}$. Consequently, the relation $\left(U_{1}, g_{1}\right) \leq\left(U_{2}, g_{2}\right)$ if $U_{1} \subset U_{2}$ is a partial order on $\mathcal{F}$.

We define the extension as follows $\mathcal{U}:=\bigcup_{(U, g) \in \mathcal{F}} U$ and the function $f: \mathcal{U} \rightarrow \mathbb{D}$ with the formula $f(\lambda):=g(\lambda)$ if $\lambda \in U$ and $(U, g) \in \mathcal{F}$. Note that the function $f$ is well defined, holomorphic, coincides with $f_{0}$ on $\triangle(0, \epsilon)$ and $|f(\lambda)|<|\lambda|, \lambda \in \mathcal{U} \backslash\{0\}$. The element $(\mathcal{U}, f)$ is a maximal element of $\mathcal{F}$. It is sufficient to show that $\mathcal{U}=\mathbb{D}$. Suppose the opposite. Take a point $\mu \in \partial \mathcal{U} \cap \mathbb{D}$ such that the ray $\{r \mu: r \in[0,1)\} \subset \mathcal{U}$. First note that $f(\mu):=\lim _{\mathcal{U}_{\ni \lambda \rightarrow \mu}} f(\lambda)$ exists and $|f(\mu)| \leq|\mu|$, as otherwise $W$ would contain over $\mu$ uncountably many points so the disc $\{\mu\} \times \mathbb{D}$ would be contained in $W$ which contradicts the irreducibility of $W$. Note also that $|f(\mu)|<|\mu|$. To see it divide $[0,1]$ into sufficiently small intervals $0=t_{0}<t_{1}<\cdots<t_{N}:=1$. Using (2.1), we get

$$
\rho(f(\mu), f(0)) \leq \sum_{j=1}^{N} \rho\left(f\left(t_{j-1} \mu\right), f\left(t_{j} \mu\right)\right)<\sum_{j=1}^{N} \rho\left(t_{j-1} \mu, t_{j} \mu\right)=\rho(\mu, 0) .
$$

We claim that $\mu \in K$. Suppose the opposite. Then $(\mu, f(\mu)) \in W_{\text {reg. The set } W_{\text {reg }}}$ is near the point $(\mu, f(\mu))$ the graph of a holomorphic function, which easily gives a strictly bigger element than $(\mathcal{U}, f)$, which contradicts its maximality.

Now we extend the function $f$ as follows. We already know that int $(\overline{\mathcal{U}})$ is $\mathbb{D}$. And now proceeding similarly as earlier, assuming that $f$ cannot be extended continuously 
to $\mathbb{D}$, we get that at some point $\mu$ from $\partial U \cap \mathbb{D}$ the cluster set of $f$ would contain uncountably many points from $\mathbb{D}$ that would force $W$ to contain $\{\mu\} \times \mathbb{D}$, a contradiction. The continuous extension of $f$ is then trivially holomorphic.

Corollary 2.4 Let $V$ be as in Theorem 2.3. Then $V$ is a Carathéodory set if and only if it is a holomorphic retract.

Proof We already know that the Carathéodory set is connected. If $V$ is not a single point, then, by Theorem 2.3, $V$ contains the graph of a complex geodesic $\mathcal{G}$ that is, up to a permutation of variables, of the form $\{(\lambda, f(\lambda)): \lambda \in \mathbb{D}\}$, where $f \in \mathcal{O}(\mathbb{D}, \mathbb{D})$. If $V$ is not equal to $\mathcal{G}$, we can assume that $(0,0) \in V$ and $f(0) \neq 0$. Note that $|f(\lambda)|<$ $|\lambda|$ for $\lambda \in \mathbb{D} \backslash\{0\}$ implies that $f(0)=0$ which contradicts our assumption, while $|f(\lambda)|>|\lambda|$ for $\lambda \in \mathbb{D}_{*}$ is impossible. Therefore, there is at least one $\mu$ (and thus uncountable many) such that $|f(\mu)|=|\mu|$. For every such $\mu$ the pair $(0,0)$ and $(\mu, f(\mu))$ is balanced. Therefore, these $\mu$ the variety $V$ contains a geodesic $\left\{\left(\lambda, \omega_{\mu} \lambda\right): \lambda \in \mathbb{D}\right\}$, where $\omega_{\mu}$ satisfies $f(\mu)=\omega_{\mu} \mu$. It is also elementary that the set of all these $\omega_{\mu}$ is uncountable. From this, we simply get that $V$ is the whole bidisc.

The equivalence of the notions of the Carathéodory set and infinitesimal Carathéodory set should hold for varieties without singular points for a reasonable class of $V \subset D$.

In the remaining part of the section, we will describe relation between Carathédory and extension sets in some classes of domains. Roughly speaking, both notions can be replaced by each other in statements of all the results that have been obtained so far. We will explain this briefly below.

Recall that a domain $D \subset \mathbb{C}^{n}$ is linearly convex if its complement is the union of complex affine hyperplanes. A domain $D$ with the smooth defining function $r$ satisfying the inequality

$$
\sum_{j, k=1}^{n} \frac{\partial^{2} r}{\partial z_{j} \partial \bar{z}_{k}}(w) X_{j} \bar{X}_{k}>\left|\sum_{j, k=1}^{n} \frac{\partial^{2} r}{\partial z_{j} \partial z_{k}}(w) X_{j} X_{k}\right|
$$

for any $w \in \partial D, X \neq 0$ lying in the complex tangent hyperplane to $\partial D$ at $w$ is called strongly linearly convex.

Remark 2.5 Using methods from this paper and those used in [15], we can get the following result. Let a domain $D$ in $\mathbb{C}^{n}$ be strictly convex or strongly linearly convex. Let $V$ be a relatively polynomially convex analytic subset of $D$. Then $V$ is a Carathéodory set if and only if it is totally geodesic.

In particular, if $D$ is the Euclidean ball $\mathbb{B}_{n}$ or if $n=2$, then any Carathéodory set $V$ is a holomorphic retract.

Remark 2.6 Except for examples described above, the extension property problem was solved fully only in a particular example of the domain

$$
\mathbb{G}_{2}:=\{(\lambda+\mu, \lambda \mu): \lambda, \mu \in \mathbb{D}\}
$$

called the symmetrized bidisc. It turns out that both notions coincide there in a reasonable class of domains. More precisely, an algebraic set $V$ in $\mathbb{G}_{2}$ is a Carathéodory 
set if and only if it has extension property. Moreover, there are one dimensional Carathéodory sets in $\mathbb{G}_{2}$ that are not complex geodesics.

To see this one needs to follow the proof in [3] to get that any Carathéodory set $V$ is either $V_{\beta}$ or $V_{\beta} \cup \Sigma, \beta \in \mathbb{D}, V_{\beta}=\{(\beta+\bar{\beta} \lambda, \lambda): \lambda \in \mathbb{D}\}$ and $\Sigma=\left\{\left(2 \lambda, \lambda^{2}\right): \lambda \in \mathbb{D}\right\}$ or there is a biholomorphic mapping $\iota: \mathbb{D} \rightarrow V$. In the latter case, $\rho\left(l^{-1}(z), \iota^{-1}(w)\right) \leq$ $c_{V}(z, w)=c_{\mathbb{G}_{2}}(z, w)$ for $z, w \in V$. Thus, for $\iota(\lambda)=z$ and $\iota(\mu)=w$, we get $\rho(\lambda, \mu) \leq$ $c_{\mathbb{G}_{2}}(\iota(\lambda), \iota(\mu)) \leq \rho(\lambda, \mu)$, which means that $\iota$ is a geodesic.

Remark 2.7 The extension problem has been studied in the tridisc in [14], where partial characterization of extension sets were obtained. One can modify arguments used there along methods exploited within the proof of Theorems 2.3 and 2.4 to get that all the assertions of the main results for extension sets in [14] are also satisfied by Carathéodory sets.

\section{Carathéodory Sets in the Tridisc_the Case of the Sets $M_{\alpha}$}

Our first and main result of this section is the proof of the fact that the sets $M_{\alpha}$-twodimensional algebraic submanifolds of $\mathbb{D}^{3}$ defined earlier-are Carathéodory sets. Moreover, the equality as in the Lempert theorem holds for them. The main result is formulated below.

\section{Theorem 3.1 The set}

$$
M_{\alpha}=\left\{z \in \mathbb{D}^{3}: \alpha_{1} z_{1}+\alpha_{2} z_{2}+\alpha_{3} z_{3}=\bar{\alpha}_{3} z_{1} z_{2}+\bar{\alpha}_{2} z_{1} z_{3}+\bar{\alpha}_{1} z_{2} z_{3}\right\}
$$

is Carathéodory. Moreover, $l_{M_{\alpha}} \equiv c_{M_{\alpha}} \equiv\left(c_{\mathbb{D}^{3}}\right)_{\mid M_{\alpha}^{2}}$.

What remains unclear for us is whether the sets $M_{\alpha}$ have the extension property. Moreover, as we shall also see later in the section, the sets $M_{\alpha}$ give rise to a construction of two-dimensional domains (denote by $D_{a, b}$ ) that are Lempert. To the best of our knowledge, the fact that the domains $D_{a, b}$ are Lempert cannot be proved by methods developed by Lempert. This makes the sets extremely interesting from that point of view-we shall address these problems at the end of this section.

The result is trivial if $M:=M_{\alpha}$ is a retract. Therefore, from now on we assume that this is not the case. In other words, the inequalities $\left|\alpha_{j_{1}}\right|+\left|\alpha_{j_{2}}\right|>\left|\alpha_{j_{3}}\right|$ are satisfied for all permutations $\left(j_{1}, j_{2}, j_{3}\right)$ of the set $\{1,2,3\}$. In the sequel, the triples $\alpha$ satisfying this property will be called the ones that satisfy the triangle inequality. Note that if the triple $\alpha$ satisfies the triangle inequality then $\alpha_{j} \neq 0, j=1,2,3$.

According to Proposition 1.6, to get the assertion it is sufficient to show that $M$ is totally geodesic. To prove it we shall first show in Lemma 3.2 that it is infinitesimally totally geodesic. Then a topological argument will finish the proof.

Using Remark 1.2, we can make the following reduction. Instead of proving the fact that $M_{\alpha}$ is Carathéodory for the fixed $\alpha$, it is sufficient to show that

$$
l_{M_{\alpha}}(0, x)=c_{M_{\alpha}}(0, x)=c_{\mathbb{D}^{3}}(0, x), \quad x \in M_{\alpha}
$$

for any $M_{\alpha}$ being not a retract.

Then the idea of the proof of the above equality is the following. First, we show the existence of a complex geodesic passing through the origin and arbitrary vector 
tangent to $M_{\alpha}$ at 0 . It is theoretically possible that not all the points from the set $M_{\alpha}$ will be achieved by such geodesics. But the use of some topological argument will provide the existence of a geodesic passing through 0 and arbitrary point of $M_{\alpha}$. At the same time, we show that left inverses to all such geodesics can be attained by one of three functions: the projections of $M$ onto one of the three axes.

We fix now $M=M_{\alpha}$ being not a retract. Note that $M$ can be written as

$$
M=\left\{z \in \mathbb{D}^{3}: z_{3}=\omega \frac{a z_{1}+b z_{2}-z_{1} z_{2}}{\bar{b} z_{1}+\bar{a} z_{2}-1}\right\},
$$

for some numbers $a, b$ such that the triple $\{a, b, 1\}$ satisfies the triangle inequality and unimodular $\omega$. Using a linear automorphism of $\mathbb{D}^{3}$, we can additionally assume that $a, b>0$ and $\omega=1$. Note that in such a case, $T_{0} M=\left\{X \in \mathbb{C}^{3}: a X_{1}+b X_{2}+X_{3}=0\right\}$.

We start the proof with the following statement.

\section{Lemma 3.2 Define}

$$
X:=\left\{\gamma=\left(\gamma_{1}, \gamma_{2}\right) \in \mathbb{C}^{2}:(\gamma, 1) \in T_{0} M \text { and }\left|\gamma_{1}\right|,\left|\gamma_{2}\right|<1\right\} .
$$

Then there are two continuous mappings

$$
(\omega, \eta)=(\omega, \eta)(\gamma): X \longrightarrow \mathbb{T}^{2}
$$

such that for any $\gamma \in \mathcal{X}$ the image of the mapping

$$
(\dagger) \quad \Phi_{\gamma}: \mathbb{D} \ni \lambda \longmapsto\left(\lambda m_{\gamma_{1}}(\omega \lambda), \lambda m_{\gamma_{2}}(\eta \lambda), \lambda\right)
$$

lies in $M\left(m_{v}(\lambda):=\frac{v-\lambda}{1-\bar{v} \lambda}, \lambda \in \mathbb{D}, v \in \mathbb{D}\right)$.

The two existing mappings $(\omega, \eta)$ are such that both components differ at each argument.

Consequently, for any $\gamma \in X$, there are two non-equivalent geodesics (i.e., having different image) passing through the pair $(0 ;(\gamma, 1))$.

We also have the "infinitesimal" version of the Lempert theorem at 0; namely, the equality $\kappa_{M}(0 ; X)=\gamma_{M}(0 ; X)$ holds for any $X \in T_{0} M$.

Remark 3.3 The set $X$ is geometrically lens (linear transformation of intersection of two discs) with exactly two points $\gamma, \tilde{\gamma}$ from the closure that lie in $\mathbb{T}^{2}$.

Proof of Lemma 3.2 Let us recall that the triple $\{a, b, 1\}$ satisfies the triangle inequality; it will be used in the sequel extensively.

Our aim is to show for any $\gamma \in \mathcal{X}$ the existence of exactly two pairs $(\omega, \eta)(\gamma) \in \mathbb{T}^{2}$ (moreover, varying continuously) such that the equality

$$
a \lambda \frac{\gamma_{1}-\omega \lambda}{1-\omega \bar{\gamma}_{1} \lambda}+b \lambda \frac{\gamma_{2}-\eta \lambda}{1-\eta \bar{\gamma}_{2} \lambda}+\lambda=a \lambda^{2} \frac{\gamma_{2}-\eta \lambda}{1-\eta \bar{\gamma}_{2} \lambda}+b \lambda^{2} \frac{\gamma_{1}-\omega \lambda}{1-\omega \bar{\gamma}_{1} \lambda}+\lambda^{2} \frac{\gamma_{1}-\omega \lambda}{1-\omega \bar{\gamma}_{1} \lambda} \frac{\gamma_{2}-\eta \lambda}{1-\eta \bar{\gamma}_{2} \lambda}
$$

holds for all $\lambda \in \mathbb{D}$. if

Keeping in mind that $\gamma \in \mathcal{X}$, we easily get that the above equality holds if and only

$$
a \omega+a \eta \bar{\gamma}_{2} \gamma_{1}+b \eta+b \omega \bar{\gamma}_{1} \gamma_{2}+\omega \bar{\gamma}_{1}+\eta \bar{\gamma}_{2}=-a \gamma_{2}-b \gamma_{1}-\gamma_{1} \gamma_{2}
$$


Keeping in mind that $\gamma \in \mathcal{X}$ (more precisely making use of the equality $a \gamma_{1}+b \gamma_{2}+$ $1=0$ ), we get that the last is equivalent to

$$
a \omega\left(1-\left|\gamma_{1}\right|^{2}\right)+b \eta\left(1-\left|\gamma_{2}\right|^{2}\right)+a \gamma_{2}+b \gamma_{1}+\gamma_{1} \gamma_{2}=0
$$

The elementary planar geometric properties show that to finish the proof it is sufficient to show that for all $\gamma \in \mathcal{X}$, the following inequalities hold

$$
\begin{aligned}
\left|a\left(1-\left|\gamma_{1}\right|^{2}\right)-b\left(1-\left|\gamma_{2}\right|^{2}\right)\right| & <\left|a \gamma_{2}+b \gamma_{1}+\gamma_{1} \gamma_{2}\right| \\
& <a\left(1-\left|\gamma_{1}\right|^{2}\right)+b\left(1-\left|\gamma_{2}\right|^{2}\right) .
\end{aligned}
$$

To prove the right inequality, we consider the function

$$
h(\gamma):=\left|a \gamma_{2}+b \gamma_{1}+\gamma_{1} \gamma_{2}\right|-a\left(1-\left|\gamma_{1}\right|^{2}\right)-b\left(1-\left|\gamma_{2}\right|^{2}\right)
$$

that is defined on a complex line $l$ containing $X$ (that is identified as a subdomain of l). The function $h$ is subharmonic as a function of a complex variable. To prove the desired inequality, it is sufficient to show that $h$ is 0 on the boundary of $X$. Take $\gamma$ from the boundary of $\mathcal{X}$. We can assume that $\left|\gamma_{2}\right|=1$. Then the elementary calculations give

$$
h(\gamma)=\left|a+b \gamma_{1} \bar{\gamma}_{2}+\gamma_{1}\right|-a\left(1-\left|\gamma_{1}\right|^{2}\right)=0 .
$$

The proof of the second inequality goes as follows. Due to symmetry, it is sufficient to show that

$$
\left|a \gamma_{2}+b \gamma_{1}+\gamma_{1} \gamma_{2}\right|+b\left(1-\left|\gamma_{2}\right|^{2}\right)>a\left(1-\left|\gamma_{1}\right|^{2}\right)
$$

Note that the left side of the above inequality is

$$
\begin{aligned}
\mid a \gamma_{2} & -\frac{b}{a}\left(b \gamma_{2}+1\right)-\frac{b \gamma_{2}+1}{a} \gamma_{2} \mid+b\left(1-\left|\gamma_{2}\right|^{2}\right) \\
& =\left|\frac{b}{a} \gamma_{2}^{2}+\frac{b}{a}+\frac{1+b^{2}-a^{2}}{a} \gamma_{2}\right|+b\left(1-\left|\gamma_{2}\right|^{2}\right) \\
& \geq \frac{b}{a}\left|\gamma_{2}-1\right|^{2}-\frac{b^{2}+2 b+1-a^{2}}{a}\left|\gamma_{2}\right|+b\left(1-\left|\gamma_{2}\right|^{2}\right) .
\end{aligned}
$$

Therefore, it is sufficient to show that for all $\gamma_{2} \in \mathbb{D}$, we have

$$
\frac{b\left|1-\gamma_{2}\right|^{2}}{a}-\frac{(b+1)^{2}-a^{2}}{a}\left|\gamma_{2}\right|+b\left(1-\left|\gamma_{2}\right|^{2}\right)>\frac{a^{2}-\left|b \gamma_{2}+1\right|^{2}}{a} .
$$

Simplifying above and then dividing by $1+b-a$, the last is equivalent to

$$
b\left|\gamma_{2}\right|^{2}+(a+1)-(b+1+a)\left|\gamma_{2}\right|>0,
$$

which is equivalent to the inequality

$$
\left(1-\left|\gamma_{2}\right|\right)\left(a+1-b\left|\gamma_{2}\right|\right)>0 \text {. }
$$

And the last inequality holds trivially.

Remark 3.4 A small modification of the proof of Lemma 3.2 gives a much wider variety of complex geodesics having the components being the Blaschke product of degree one or two. The idea is the following. For $|\omega|=1, \gamma \in \mathbb{D}$, we put

$$
\varphi_{\omega, \gamma}(\lambda)=\left(\lambda \frac{\gamma-\omega \lambda}{1-\bar{\gamma} \omega \lambda}=: \lambda \psi(\lambda), \frac{a \lambda+b \lambda \psi(\lambda)-\lambda^{2} \psi(\lambda)}{b \lambda+a \lambda \psi(\lambda)-1}, \lambda\right) .
$$


Our aim will be to find $\omega$ so that the above mapping lies entirely in $M$, and the second component is the Blaschke product of degree two.

Recall that the Schur algorithm gives that the square polynomial $A \lambda^{2}+B \lambda+C$ has both roots outside $\overline{\mathbb{D}}$ if and only if $|C|>|A|$ and $|C|^{2}-|A|^{2}>|B \bar{C}-A \bar{B}|$. Applying this property to our function (coming from the denominator of the second component), we get that the following inequality for $\omega$ that has to be satisfied

$$
b^{2}-|a \gamma+1|^{2}>\left|-a b\left(1-|\gamma|^{2}\right)+\omega\left(a \bar{\gamma}^{2}+\left(a^{2}-b^{2}+1\right) \bar{\gamma}+a\right)\right| .
$$

The above inequality is equivalent to the following one:

$$
b\left(1-\left|\gamma_{2}\right|^{2}\right)>\left|a\left(1-\left|\gamma_{1}\right|^{2}\right)-\bar{\omega}\left(a \gamma_{2}+b \gamma_{1}+\gamma_{1} \gamma_{2}\right)\right|,
$$

where $\gamma_{1}:=\gamma, \gamma_{2}:=-\frac{a \gamma_{1}+1}{b}$. And now we make use of the calculations conducted in the proof of the previous lemma-to see that the above inequality holds for $|\omega|=1$ lying in a non-empty open arc.

The above reasoning gives the following property for the variety $M$. If only $X \in T_{0} M$ is such that $\left|X_{1}\right|>\left|X_{j}\right|, j=2,3$, we get following functions

$$
\mathbb{D} \ni \lambda \longmapsto\left(\lambda, \lambda m_{\eta}(\omega \lambda), f_{3}(\lambda)\right) \in M,
$$

where $\eta$ is suitably chosen and $|\omega|=1$ can be chosen from some non-empty arc.

The above result gives an example of a two-dimensional domain for which the infinitesimal version of the Lempert theorem holds. Namely, let

$$
D_{a, b}:=\left\{z \in \mathbb{D}^{2}:\left|F_{a, b}\left(z_{1}, z_{2}\right)\right|<1\right\},
$$

where $F_{a, b}\left(z_{1}, z_{2}\right):=\frac{a z_{1}+b z_{2}-z_{1} z_{2}}{a z_{2}+b z_{1}-1}$.

Corollary 3.5 The following equality holds:

$$
\kappa_{D_{a, b}} \equiv \gamma_{D_{a, b}} .
$$

In the special case of the origin, we have the following formula:

$$
\kappa_{D_{a, b}}(0 ; X)=\max \left\{\left|X_{1}\right|,\left|X_{2}\right|,\left|a X_{1}+b X_{2}\right|\right\}, X \in \mathbb{C}^{2}, z \in M .
$$

Having proven Lemma 3.2, we will show that $M$ is the Carathéodory set. As we already announced, the proof will be a consequence of Lemma 3.2 and a topological argument.

Proof of Theorem 3.1 We show that

$$
c_{M}(0, z)=l_{M}(0, z)=\max \left\{\rho\left(0, z_{j}\right): j=1,2,3\right\} .
$$

Below we use the notation as in Lemma 3.2. To show the above, it is sufficient to show that for any $x \in \mathbb{D}_{*}$ and any $\left(\lambda_{1}, \lambda_{2}\right) \in M_{x}$, where

$$
M_{x}:=\left\{\left(\lambda_{1}, \lambda_{2}\right) \in \mathbb{D}^{2}:\left(\lambda_{1} x, \lambda_{2} x, x\right) \in M\right\},
$$

there is a $\gamma \in X$ such that $\Phi_{\gamma}(x)=\left(\lambda_{1} x, \lambda_{2} x, x\right)$. Denote $\Psi_{\gamma}(x):=\frac{\left(\Phi_{\gamma}(x)\right)_{1,2}}{x}$. To finish the proof, it is sufficient to show that the function

$$
\psi_{x}: X \ni \gamma \longmapsto \Psi_{\gamma}(x) \in M_{x}
$$


is onto. The properties of the functions $(\omega, \eta)$ imply that $\psi_{x}$ is not only continuous, but it also extends continuously onto the closure of $\mathcal{X}$. Moreover, if $\left|\gamma_{j}\right|=1$ is such that $\gamma \in \bar{X}$, then the extension satisfies the equality $\left(\psi_{x}(\gamma)\right)_{j}=\gamma_{j}$. Both $X$ and $M_{x}$ are simply connected two-dimensional surfaces bounded by union of two arcs and such that $\psi_{x}$ is a homeomorphism between the boundaries. Consequently, $\psi_{x}$ is onto.

As a direct consequence of the theorem, we get the equality of invariant functions in a class of two-dimensional domains.

\section{Theorem 3.6 The domain $D_{a, b}$ is a Lempert domain.}

Remark 3.7 Note that if the triple $\{1, a, b\}$ satisfies the triangle inequality, then the domain $D_{a, b}$ is never linearly convex. Actually, to see this take an arbitrary point $w \in \partial D_{a, b} \cap \mathbb{D}^{2}$. Then $\left|F_{a, b}(w)\right|=1$. Assuming the linear convexity, we find a vector $v \in \mathbb{C}^{2} \backslash\{0\}$ such that $\left|F_{a, b}(w+\lambda v)\right| \geq 1$ for $\lambda$ close to zero. The minimum principle for holomorphic functions implies that $F_{a, b}(w+\lambda v)$ equals some unimodular constant for $\lambda$ close to 0 , which in view of the explicit formula for $F_{a, b}$ easily implies that either $v_{1}$ or $v_{2}$ is zero. Assume that $v_{1}=0$. Then the function $\lambda \rightarrow F_{a, b}(w+\lambda v)$ is a homography; the elementary calculations give that it is constant precisely when

$$
b w_{1}^{2}-\left(b^{2}+1-a^{2}\right) w_{1}+b=0 .
$$

But the above equation is satisfied only for $\left|w_{1}\right|=1$ (use the triangle inequality for the triple $\{a, b, 1\}$ to see that the roots of the above square equation are not reals), which gives the contradiction.

Remark 3.8 As we saw, the Lempert Theorem holds for the bounded hyperconvex domain $D_{a, b}$ that is not linearly convex. It would be desirable to decide whether the domain $D_{a, b}$ is not biholomorphic to a convex domain. Note that the domains $D_{a, b}$ are the next candidates for examples of that kind. Perhaps from the point of view of the Lempert Theory the domains $D_{a, b}$ have much less nice properties than the existing examples of that type (the symmetrized bidisc and tetrablock). In any case, it seems reasonable that an effort should be undertaken to understand better the function geometric properties of that class of domains.

Remark 3.9 Let us underline once more that a study of a class of two-dimensional submanifolds of the tridisc that appeared in the study of the extension property in [14] led not only to introducing a new property that seems to be better suited in the study of the extension property (Carathéodory sets), but also provides examples of domains interesting for the geometric function theory.

\section{Universal Sets for the (Infinitesimal) Carathéodory Extremal Problem}

In [2], the authors introduced the notion of universal sets for the Carathéodory extremal problem concentrating mainly on the problem in the symmetrized bidisc. In this section, we concentrate on that topic presenting results on the domains admitting finite universal sets. 
Let $D$ be a domain in $\mathbb{C}^{n}$. We say that $\mathcal{C} \subset \mathcal{O}(D . \mathbb{D})$ is a universal set for the Carathéodory (resp. for the infinitesimal Carathéodory) extremal problem if for any $w, z \in D, w \neq z$ (resp. $w \in D, X \in \mathbb{C}^{n}, X \neq 0$ ), there is an $F \in \mathcal{C}$ such that $c_{D}(w, z)=\rho(F(w), F(z))\left(\right.$ resp. $\left.\gamma_{D}(w ; X)=\gamma_{\mathbb{D}}\left(F(w) ; F^{\prime}(w)(X)\right)\right)$.

Remark 4.1 Assume that $D$ is $c$-hyperbolic and $\gamma$-hyperbolic (i.e., $c_{D}(w, z)>0$ for all $w, z \in D, w \neq z$ and $\left.\gamma_{D}(w ; X)>0, w \in D, X \in \mathbb{C}^{n} \backslash\{0\}\right)$. If $\mathcal{C}$ is a universal set for the Carathéodory extremal problem, then $\mathcal{C}$ is also a universal set for the infinitesimal Carathéodory extremal problem.

As to the problem of the existence of finite universal sets, we present a result on one-dimensional domains and show that the domains $D_{a, b}$ introduced in Section 3 are examples showing that the situation in dimension one and two are completely different. We also simplify and extend results on characterization of domains with finite universal sets presented in [2].

Recall that the domain $D$ is c-finitely compact if the Carathéodory balls $\{z \in D$ : $\left.c_{D}(w, z)<r\right\}$ are relatively compact in $D$ for all $w \in D, r>0$.

\subsection{Planar Domains with Minimal Universal Sets}

Recall that for planar domains, $c$-hyperbolicity is equivalent to $\gamma$-hyperbolicity and this is equivalent to the existence of a non-constant bounded holomorphic function (see e.g., [12]).

The infinitesimal version of the proposition below is the content of [9, Theorem 1]. The non-infinitesimal case can be obtained along the same lines.

Proposition 4.2 ( $c f .[9$, Theorem 1]) Let D be a planar domain. Then Carathéodory extremals and infinitesimal Carathéodory extremals are uniquely determined which means that up to automorphisms of the unit disc for any $w \neq z$ (resp. $w \in D)$, there is only one $F \in \mathcal{O}(D, \mathbb{D})$ such that

$$
\begin{aligned}
c_{D}(w, z) & =\rho(F(w), F(z)), \\
\left(\text { resp. } \gamma_{D}(w ; 1)\right. & \left.=\gamma_{\mathbb{D}}\left(F(w) ; F^{\prime}(w)\right)\right) .
\end{aligned}
$$

We show that in the case of the planar domains, the existence of finite universal sets makes the domain (under some evident assumptions) the unit disc that is contained in the following theorem.

Theorem 4.3 Let $D$ be a domain in $\mathbb{C}$ that has a finite universal set for the infinitesimal Carathéodory problem. Then $D$ has a universal set consisting of one element. In particular, if $D$ is additionally c-finitely compact, then it is biholomorphic to the unit disc.

Proof Without loss of generality, we can assume that $D$ is $\gamma$-hyperbolic. Let $\mathcal{C}=$ $\left\{\Phi_{1}, \ldots, \Phi_{N}\right\}$ be a minimal finite universal set for the infinitesimal Carathéodory extremal problem. It is sufficient to show that $N=1$. Suppose that $N \geq 2$. Denote $V:=\Phi(D)$. The uniqueness of $\gamma$-extremals and the minimality of $\mathcal{C}$ imply that for any $z \in D$, there is a $j$ such that

$$
\gamma_{\mathbb{D}}\left(\Phi_{j}(z) ; \Phi_{j}^{\prime}(z)\right)>\max \left\{\gamma_{\mathbb{D}}\left(\Phi_{k}(z) ; \Phi_{k}^{\prime}(z)\right), k \neq j\right\} .
$$


On the other hand, the minimality of the set $\mathcal{C}$ implies that for any $1 \leq k \leq N$ there is a $z \in D$ such that

$$
\gamma_{\mathbb{D}}\left(\Phi_{k}(z) ; \Phi_{k}^{\prime}(z)\right)>\max \left\{\gamma_{\mathbb{D}}\left(\Phi_{j}(z) ; \Phi_{j}^{\prime}(z)\right): j \neq k\right\} .
$$

A standard connectivity argument shows, however, that both statements cannot hold simultaneously.

\subsection{Finite Universal Sets Induce Embeddings into Polydiscs}

Below we generalize [2, Theorem 2.3] with a simpler proof.

Theorem 4.4 Let $D \subset \mathbb{C}^{n}$ be c-hyperbolic and $\gamma$-hyperbolic. Assume additionally that $\mathcal{C}=\left\{\Phi_{1}, \ldots, \Phi_{N}\right\}$ is a universal set for the Carathéodory extremal problem. Then the mapping

$$
\Phi:=\left(\Phi_{1}, \ldots, \Phi_{N}\right): D \longrightarrow \mathbb{D}^{N}
$$

is a holomorphic embedding. In particular, $N \geq n$ and $\Phi(D)$ is a connected complex submanifold of dimension $n$.

Moreover, $\Phi(D)$ is a Carathéodory set. In particular, we get

$$
\begin{aligned}
c_{\Phi(D)}(w, z) & =\max \left\{\rho\left(\Phi_{j}(w), \Phi_{j}(z)\right): j=1, \ldots, N\right\}, \\
\gamma_{\Phi(D)}\left(\Phi(w) ; \Phi^{\prime}(w)(X)\right) & =\max \left\{\gamma_{\mathbb{D}}\left(\Phi_{j}(w) ; \Phi_{j}^{\prime}(w)(X): j=1, \ldots, N\right\}\right.
\end{aligned}
$$

$w, z \in D, X \in \mathbb{C}^{n}$.

If $D$ is additionally c-finitely compact, then $\Phi$ is proper so in the case $n=N$ we get that $\Phi(D)=\mathbb{D}^{n}$.

Proof The definition of the universal Carathéodory set gives

$$
\begin{aligned}
c_{D}(w, z) & =\max \left\{\rho\left(\Phi_{j}(w), \Phi_{j}(z)\right): j=1, \ldots, N\right\} \\
& =c_{\mathbb{D}^{N}}(\Phi(w), \Phi(z)), w, z \in D .
\end{aligned}
$$

Let $w, z \in D$ be such that $\Phi(w)=\Phi(z)$. Then $c_{D}(w, z)=0$ and the $c$-hyperbolicity implies that $w=z$. Therefore, $\Phi$ is injective. Similarly, because of the fact that $\left\{\Phi_{1}, \ldots, \Phi_{n}\right\}$ is a universal set for the infinitesimal Carathéodory extremal problem, we get

$$
\begin{aligned}
\gamma_{D}(w ; X) & =\max \left\{\gamma_{\mathbb{D}}\left(\Phi_{j}(w) ; \Phi_{j}^{\prime}(w)(X)\right): j=1, \ldots, N\right\} \\
& \left.=\gamma_{\mathbb{D}^{N}}\left(\Phi(w) ; \Phi^{\prime}(w)(X)\right)\right\}, w \in D, X \in \mathbb{C}^{n} .
\end{aligned}
$$

Since $D$ is $\gamma$-hyperbolic, we get that the rank of $\Phi^{\prime}(w)$ is $n$.

We prove that $V:=\Phi(D)$ is a Carathéodory set. This can be seen as follows:

$$
\begin{aligned}
\mathcal{c}_{\mathbb{D}^{N}}(\Phi(w), \Phi(z))=\max \left\{\rho\left(\Phi_{j}(w), \Phi_{j}(z)\right): j=1, \ldots, N\right\}= \\
c_{D}(w, z)=c_{\Phi(D)}(\Phi(w), \Phi(z)) \geq c_{\mathbb{D}^{N}}(\Phi(w), \Phi(z)) .
\end{aligned}
$$

Assume that $D$ is additionally $c$-finitely compact. We show below that $\Phi$ is proper. Fix $w \in D$ and let $\left(z^{k}\right)_{k}$ have no accumulation point in $D$. Then the equality

$$
c_{\mathbb{D}^{N}}\left(\Phi(w), \Phi\left(z^{k}\right)\right)=c_{D}\left(w, z^{k}\right) \longrightarrow \infty
$$


implies that $\left(\Phi\left(z^{k}\right)\right)_{k}$ has no accumulation point in $\mathbb{D}^{N}$, which gives the desired properness of $\Phi$.

\subsection{Not Only Polydisc Admits Finite Universal Sets for the Carathéodory Extremal Problem in Higher Dimension}

It is shown in [2] that the projections are contained in any universal Carathéodory set of the bidisc. It turns out that under evident assumptions domains $D_{a, b}$ have similar property-the three functions defining $D_{a, b}$ must lie in any universal Carathéodory set. Moreover, the domains $D_{a, b}$ are examples of (very nice, for instance $c$-finitely compact) domains that admit the finite universal Carathéodory sets and but are still not the bidisc. This shows that the situation in the case $n=1$ differs from the case of bigger $n$. The fact that $D_{a, b}$ is not biholomorphic to the bidisc follows for instance from the fact that the indicatrix of $D_{a, b}$ at 0 is the domain (see Corollary 3.5)

$$
\left\{X \in \mathbb{C}^{2}: \kappa_{D_{a, b}}(0 ; X)<1\right\}=\left\{X \in \mathbb{C}^{2}: \max \left\{\left|X_{1}\right|,\left|X_{2}\right|,\left|a X_{1}+b X_{2}\right|\right\}<1\right\},
$$

which is not linearly isomorphic to the bidisc under the assumption that the triple $\{a, b, 1\}$ satisfies the triangle inequality.

Example 4.5 Recall that Agler, Lykova, and Young remarked that in the bidisc any universal set for the Carathéodory problem must contain (up to an automorphism) both projections. Similar property holds for the domains $D_{a, b}$. More precisely, if the triple $\{a, b, 1\}$ satisfies the triangle inequality, then the universal set for the Carathéodory extremal problem for the domain

$$
D_{a, b}=\left\{z \in \mathbb{D}^{2}:\left|a z_{1}+b z_{2}-z_{1} z_{2}\right|<\left|b z_{1}+a z_{2}-1\right|\right\}
$$

contains (up to automorphisms) three functions:

$$
z \longmapsto z_{1}, \quad z \longmapsto z_{2} \quad \text { and } \quad z \longmapsto \frac{a z_{1}+b z_{2}-z_{1} z_{2}}{b z_{1}+a z_{2}-1} .
$$

To show the above, note that it is sufficient to see that any of the functions $z_{j}$ (up to an automorphism), $j=1,2,3$, must belong to a universal set for the Carathéodory problem in $M_{\alpha}$ being not a retract. Take such a variety. Consider the functions as in Remark 3.4

$$
\mathbb{D} \ni \lambda \longmapsto\left(\lambda, \lambda m_{\eta}(\omega \lambda), f_{3}(\lambda)\right) \in M,
$$

where $\eta$ is fixed and $\omega$ is from some non-empty arc. Then one of the left inverses (call it $F$ ) of the function for the fixed $\omega$ is a left inverse for all $\omega$ from the given arc. Consequently, the function $F$ depends only on the first variable and this equals $z_{1}$.

Remark 4.6 It follows from [14] that any subdomain of $\mathbb{C}^{2}$ that has a universal set composing of three elements is biholomorphic to a submanifold of $\mathbb{D}^{3}$ that is a graph of a holomorphic function for each choice of the coordinates. In particular, it is biholomorphic to a domain of the form $D=\left\{\left(z_{1}, z_{2}\right) \in \mathbb{D}^{2}: h\left(z_{1}, z_{2}\right)<1\right\}$, where $h$ is a holomorphic function such that $z_{1} \mapsto h\left(z_{1}, z_{2}\right)$ (respectively $z_{2} \mapsto h\left(z_{1}, z_{2}\right)$ ) is injective for every $z_{2} \in \mathbb{D}$ (resp. $z_{1} \in \mathbb{D}$ ). Some other properties of $h$ were obtained in [14]. Recall that the results in [14] are stated with the additional assumption of polynomial 
convexity. However, here they can be dropped out. This forces two natural questions. The first one is if any domain with a 3-element (or finite) universal set comes from the variety $M_{\alpha}$.

The second one is more particular; namely, whether any domain having finite universal set is a Lempert domain.

\section{Universal Sets for the Carathéodory Extremal Problem for the Unit Ball $\mathbb{B}_{2}$}

In the last section, we make some remarks on the universal sets in the unit ball. The results presented can be seen as the starting point for the further study of a possible structure of (in some at the moment not well determined sense) small universal sets for the Carathéodory extremal problem.

We start with an easy observation that the unit ball $\mathbb{B}_{n}, n>1$, does not have a finite universal set for the Carathéodory extremal problem.

Proposition 5.1 The unit ball $\mathbb{B}_{n}, n>1$, does not have a finite universal set for the Carathéodory problem.

Proof This is a direct consequence of [17, Lemma 5], which states the following. Any two different complex geodesics of the ball passing through 0 have different (up to automorphisms of the unit disc) left inverses.

Remark 5.2 The result on the existence of many complex geodesics passing through 0 in the symmetrized bidisc and tetrablock that admit only one (up to an automorphism of the unit disc) left inverse can be found in [16]. The fact that the symmetrized bidisc does not have a finite universal set follows from [2, Theorem 3.1]. Consequently, the same holds for the tetrablock.

The most standard and natural procedure producing a class of the universal set for the Carathéodory extremal problem in the unit ball $\mathcal{B}_{n}$ is the following. As the unit ball is an example of a Lempert domain, both notions, extremals and infinitesimal Carathéodory extremals coincide. Moreover, the extremals are precisely the ones being the left inverses to complex geodesic, which in turn are parametrizations of portions of complex lines lying in the unit ball. To produce an extremal to one of the complex geodesics (represented by $l \cap \mathbb{B}_{n}$ ), we can proceed as follows. Let $a \in l \cap \mathbb{B}_{n}$ be the point of the minimal norm. Let $\Phi_{a}$ be the automorphism of the unit ball such that $\Phi_{a}(a)=0, \Phi_{a}(0)=a$ : recall that

$$
\Phi_{a}(z):=. \frac{\sqrt{1-\|a\|^{2}}\left(\langle z, a\rangle a-\|a\|^{2} z\right)-\langle z, a\rangle a+\|a\|^{2} a}{\|a\|^{2}(1-\langle z, a\rangle)}, \quad z \in \mathbb{B}_{n}
$$

for all $a \in \mathbb{B}_{n} \backslash\{0\}$ and $\Phi_{0}:=\operatorname{id}_{\mathbb{B}_{n}}$.

Now we can apply the unitary mapping $U$ such that $U\left(\Phi_{a}\left(l \cap \mathbb{B}_{n}\right)\right)=\mathbb{D} \times\{0\}^{n-1}$. And now the mapping $\Psi_{l}:=U_{1} \circ \Phi_{a}$ is one of possible Carathéodory extremals for the points from the geodesic $l$. 
In the simplest case of $n=2$, we can apply the above method and we see that the universal set for the Carathéodory extremal problem can be chosen in the following way:

$$
\begin{aligned}
\mathcal{C}:=\left\{\frac{\sqrt{1-\|a\|^{2}}\left(\bar{a}_{1} z_{2}-\bar{a}_{2} z_{1}\right)}{\|a\|\left(1-\left(\bar{a}_{1} z_{1}+\bar{a}_{2} z_{2}\right)\right)}: a=\left(a_{1}, a_{2}\right) \in \mathbb{B}_{2}\right\} \cup \\
\left\{a_{1} z_{1}+a_{2} z_{2}: a_{1} \geq 0,\left|a_{2}\right|^{2}=1-a_{1}^{2}\right\} .
\end{aligned}
$$

Let us make one more remark on the properties of the above construction. It follows directly from the construction of $\Psi_{l}$ that it is a rational mapping that is holomorphic on a neighborhood of $\overline{\mathbb{B}_{n}}$ and $\Psi_{l}^{-1}(\partial \mathbb{D}) \cap \overline{\mathbb{B}_{n}}=l \cap \partial \mathbb{B}_{n}$. In other words, the universal set for the Carathéodory extremal problem $\mathcal{C}$ just defined is parametrized by complex lines $l$ intersecting $\mathbb{B}_{n}$ and it is minimal in the sense that no proper subset of $\mathcal{C}$ is a universal set. Moreover, any extremal mapping is a left inverse to the unique geodesic.

It is natural to see whether one could define a class of Carathéodory extremals that could be extremals for a wider variety of geodesics, which would then yield a universal set being "smaller" than the one produced above. Following the construction of extremals from a recent paper [17], we will get the desired class of functions below. We restrict ourselves for the dimension $n=2$.

Let

$$
F\left(z_{1}, z_{2}\right):=\frac{2 z_{1}\left(1-z_{1}\right)-z_{2}^{2}}{2\left(1-z_{1}\right)-z_{2}^{2}}, \quad z \in \mathbb{B}_{2} .
$$

Recall that $F \in \mathcal{O}\left(\mathbb{B}_{2}, \mathbb{D}\right)$ and $F\left(z_{1}, 0\right)=z_{1}, z_{1} \in \mathbb{D}$ (see [17]).

Remark 5.3 Note that the mapping $F$ just defined assumes the value of absolute value one on a bigger portion of $\partial \mathbb{B}_{2}$ than that of the most natural form of extremal mappings ( $\Psi_{l}$ from the previous section). Actually, note that elementary calculations give the property that for $z \in \partial \mathbb{B}_{2}$ the equality $|F(z)|=1$ if and only if

$$
\operatorname{Im}\left(z_{2}\left(1-\bar{z}_{1}\right)\right)=0 .
$$

The above property (fact that the absolute value equal to one is assumed at twodimensional subset of $\partial \mathbb{B}_{2}$ suggests that the function $F$ can be a left inverse to a onedimensional family of complex geodesics). And this is really the case, as the next observation shows.

We claim that for any $t \in \mathbb{R}$, the mapping $F$ is the left inverse to the mapping (complex geodesic in $\mathbb{B}_{2}$ )

$$
f_{t}(\lambda):=\left(\frac{t^{2}+\lambda}{1+t^{2}}, \frac{t(\lambda-1)}{1+t^{2}}\right), \quad \lambda \in \mathbb{D} .
$$

Recall that $\left(c_{D}^{*}:=\operatorname{arctanh} c_{D}\right)$

$$
c_{\mathbb{B}_{2}}^{*}(w, z)=\sqrt{1-\frac{\left(1-\|w\|^{2}\right)\left(1-\|z\|^{2}\right)}{|1-\langle w, z\rangle|^{2}}}, \quad w, z \in \mathbb{B}_{2} .
$$

It is elementary then to check that $f_{t}(\mathbb{D}) \subset \mathbb{B}_{2}$. It is therefore sufficient to show that

$$
c_{\mathbb{B}_{2}}\left(F\left(f_{t}\left(\lambda_{1}\right), F\left(f_{t}\left(\lambda_{2}\right)\right)\right)=p\left(\lambda_{1}, \lambda_{2}\right), \quad \lambda_{1}, \lambda_{2} \in \mathbb{D},\right.
$$


which follows directly from the formula for the Carathéodory distance for the unit ball and the Poincaré distance.

Acknowledgments The authors wanted to thank the anonymous referee for comments and corrections that improved the shape of the paper.

\section{References}

[1] A. A. Abouhajar, M. C. White, and N. J. Young, A Schwarz lemma for a domain related to mu-synthesis. J. Geom. Anal. 17(2007), 717-750. https://doi.org/10.1007/BF02937435

[2] J. Agler, Z. Lykova, and N. J. Young, Characterizations of some domains via Carathéodory extremals. J. Geom. Anal. 29(2019), 3039-3054. https://doi.org/10.1007/s12220-018-0059-6

[3] J. Agler, Z. Lykova, and N. J. Young, Geodesics, retracts, and the norm-preserving extension property in the symmetrized bidisc. Mem. Amer. Math. Soc. 258(2019), no. 1242. https://doi.org/10.1090/memo/1242

[4] J. Agler and J. E. McCarthy, Norm preserving extensions of holomorphic functions from subvarieties of the bidisk. Ann. of Math. 157(2003), 289-312. https://doi.org/10.4007/annals.2003.157.289

[5] J. Agler and N. J. Young, The hyperbolic geometry of the symmetrized bidisc. J. Geom. Anal. 14(2004), no. 3, 375-403. https://doi.org/10.1007/BF02922097

[6] T. Bhattacharyya and H. Sau, Holomorphic functions on the symmetrized bidisk-realization, interpolation and extension. J. Funct. Anal. 274(2018), no. 2, 504-524. https://doi.org/10.1016/j.jfa.2017.09.013

[7] C. Costara, The symmetrized bidisc and Lempert's theorem. Bull. Lond. Math. Soc. 36(2004), no. 5, 656-662. https://doi.org/10.1112/S0024609304003200

[8] A. Edigarian, Ł. Kosiński, and W. Zwonek, The Lempert theorem and the tetrablock. J. Geom. Anal. 23(2013), no. 4, 1818-1831. https://doi.org/10.1007/s12220-012-9308-2

[9] S. D. Fisher, On Schwarz's lemma and inner functions. Trans. Amer. Math. Soc. 138(1969), 229-240. https://doi.org/10.2307/1994911

[10] K. Guo, H. Huang, and K. Wang, Retracts in polydisk and analytic varieties with the $H^{\infty}$-extension property. J. Geom. Anal. 18(2008), 148-171. https://doi.org/10.1007/s12220-007-9005-8

[11] L. F. Heath and T. J. Suffridge, Holomorphic retracts in complex n-space. Illinois J. Math. 25(1981), 125-135.

[12] M. Jarnicki and P. Pflug, Invariant distances and metrics in complex analysis. Second extended ed., De Gruyter Expositions in Mathematics, 9, Walter de Gruyter GmbH \& Co. KG, Berlin, 2013.

[13] Ł. Kosiński, Three-point Nevanlinna-Pick problem in the polydisc. Proc. Lond. Math. Soc. 111(2015), 887-910. https://doi.org/10.1112/plms/pdv045

[14] Ł. Kosiński and J. E. McCarthy, Extensions of bounded holomorphic functions on the tridisc. Rev. Mat. Iberoam. (2019).

[15] Ł. Kosiński and J. E. McCarthy, Norm preserving extensions of bounded holomorphic functions. Trans. Amer. Math. Soc. 371(2019), 7243-7257. https://doi.org/10.1090/tran/7597

[16] Ł. Kosiński and W. Zwonek, Nevanlinna-Pick problem and uniqueness of left inverses in convex domains, symmetrized bidisc and tetrablock. J. Geom. Anal. 26(2016), 1863-1890. https://doi.org/10.1007/s12220-015-9611-9

[17] Ł. Kosiński and W. Zwonek, Nevanlinna-Pick interpolation problem in the ball. Trans. Amer. Math. Soc. 370(2018), 3931-3947. https://doi.org/10.1090/tran/7063

[18] L. Lempert, La métrique de Kobayashi et la représentation des domaines sur la boule. Bull. Soc. Math. France 109(1981), no. 4, 427-474.

[19] L. Lempert, Holomorphic retracts and intrinsic metrics in convex domains. Anal. Math. 8(1982), no. 4, 257-261. https://doi.org/10.1007/BF02201775

[20] K. Maciaszek, On polynomial extension property in N-disc. Complex Anal. Oper. Theory 13(2019), 2771-2780. https://doi.org/10.1007/s11785-019-00912-3

[21] P. Pflug and W. Zwonek, Exhausting domains of the symmetrized bidisc. Ark. Mat. 50(2012), 397-402. https://doi.org/10.1007/s11512-011-0153-5

[22] W. Rudin, Function theory in polydiscs. W. A. Benjamin, New York-Amsterdam, 1969.

Institute of Mathematics, Faculty of Mathematics and Computer Science, Jagiellonian University,

Łojasiewicza 6, 30-348 Kraków, Poland

e-mail: lukasz.kosinski@uj.edu.pl wlodzimierz.zwonek@uj.edu.pl 